\title{
Behind the hunt for the Higgs boson
}

Tension is building on both sides of the Atlantic in the race to find the Higgs boson, the elusive particle predicted by physicists' understanding of mass.

Scientists working at the Tevatron particle collider at Fermilab in Batavia, Illinois, welcomed the new year by measuring the mass of another subatomic particle, the W boson. This finding lowers the expected mass of the Higgs, bringing it further into the range in which the Tevatron might be able to find it.

Meanwhile, the Tevatron's more powerful competitor, the Large Hadron Collider (LHC), is entering its final stages of construction at CERN, the European particle-physics lab near Geneva in Switzerland. If the Higgs exists, the LHC should find it, but the spark of a chance that the Tevatron might get there first is energizing both teams. "We are pulling out all the stops," says Mark Lancaster, one of around 700 scientists working on the Collider Detector at Fermilab (CDF) - an instrument that collects and analyses debris from protonantiproton collisions in the Tevatron.

Jim Virdee at CERN is the spokesman for one of the LHC's experiments, the Compact Muon Spectrometer. "We are aware of this competition," he says. "It makes us make sure the experiments and the accelerator are ready on time." The enormous parts of the spectrometer are currently being lowered delicately into place in the LHC's tunnels.

The Higgs is thought to be a missing piece of the standard model of particle physics, which lumps together what is known about the fundamental forces and particles. Theories that extend the standard model without the Higgs tend to be more complex than those that include it - leaving physicists hopeful that it will turn up.

Now that estimates of the Higgs' mass have shrunk, the odds have improved that the Tevatron will be able to detect it. In 2004, the upper bound of the boson's mass hovered around 250 gigaelectron volts $(\mathrm{GeV})$, which was beyond the Tevatron's upper limit of about $170 \mathrm{GeV}$.

But estimates of the Higgs' mass are based on the mass of other particles, such as the top quark and Wboson, and updates in these measurements have since dragged the upper limit on the Higgs down. Most recently, on 8 January, the CDF measured the mass of the $\mathrm{W}$ boson more precisely than ever before, at $80.413 \mathrm{GeV}$.

That figure is consistent with previous results, but towards the upper end of the range, and brings the upper limit for the Higgs down to $153 \mathrm{GeV}$ from $166 \mathrm{GeV}$. The Large Electron-Positron (LEP) collider, which CERN hosted until it was shut down in 2000 , ruled out masses of up to $114.4 \mathrm{GeV}$ - leaving just a small window still to explore (see Graphic).

At the same time, the Tevatron has overcome some of the performance shortfalls that had dampened hopes of finding it at any mass. So researchers there, despite the threat of budget cuts forcing a temporary closure, are allowing themselves some optimism. "We have got about three years," says Lancaster. The Tevatron will run until 2009 and, although the LHC is due to switch on for a warm-up run in November, it will not start to collect data seriously until spring 2008.

Ironically, the Tevatron's next Higgs announcement might be about not finding it. Just before LEP closed to make way for the LHC, it saw hints of a Higgs at around $115 \mathrm{GeV}$. The thought that the Higgs had narrowlyescaped their grasp has haunted CERN's physicists ever since, but the Tevatron is on the verge of having enough data to rule out a particle of that mass. Unless something emerges in the next few months, the LEP ghost will be laid to rest. "I would say that we would be able to rule out this hint from LEP before the LHC gets going in earnest," says Lancaster.

Jenny Hogan 